\title{
A Subclass of close-to-star functions
}

\author{
C.Selvaraj ${ }^{1}$ and S. Logu ${ }^{2 *}$
}

\begin{abstract}
In this paper, we generalise the concept of close-to-star functions by considering a subclass $\mathscr{K}_{s}^{*}(\alpha, \beta)$ of normalized analytic functions in the unit disk $\mathbb{U}=\{z \in \mathbb{C}:|z|<1\}$ such that for some starlike function $g(z)=z+b_{2} z^{2}+\ldots$

$$
\frac{z f^{\prime}(z)}{g(z)} \prec \frac{1+(2 \alpha-1) \beta z}{1+\beta z}, \quad 0 \leq \alpha<1, \quad 0<\beta \leq 1 .
$$

We obtain results on sharp coefficient estimates, growth and distortion theorems. .

\section{Keywords}

Analytic functions, coefficient estimates,starlike function.

AMS Subject Classification: 30c45.

${ }^{1}$ Department of Mathematics, Presidency College, Chennai, India.

${ }^{2}$ Department of Mathematics, Easwari Engineering College, Ramapuram, Chennai - 600 089, Tamil Nadu, India.

*Corresponding author: ${ }^{\text {* }}$ pamc9439@yahoo.co.in; ${ }^{2}$ logumaths123@gmail.com

\section{Contents}

1 Introduction and Definitions 290

2 Preliminaries 291

3

4

COEFFICIENT BOUNDS 291

4 Growth and distortion theorems 292

References .294

\section{Introduction and Definitions}

Let $\mathscr{A}$ be the normalized class of regular functions

$$
f(z)=z+a_{2} z^{2}+a_{3} z^{3}+\ldots
$$

which are univalent in the unit disk $\mathbb{U}=\{z \in \mathbb{C}:|z|<1\}$ and normalized by the conditions $f(0)=f^{\prime}(0)-1=0$.

If $f \in \mathscr{A}$ and satisfies $\mathfrak{R}\left\{\frac{z f^{\prime}(z)}{f(z)}\right\}>\alpha, \quad \forall z \in \mathbb{U}$, then $f(z)$ is said to be a starlike functions of order $\alpha$. We use $\mathscr{S}^{*}(\alpha)$ to denote the class of starlike function of order $\alpha$. On putting $\alpha=0$, the class of functions is denoted by $\mathscr{S}^{*}$. A function $f(z)$ belonging to $\mathscr{A}$ is said to be convex if and only if $\mathfrak{R}\left\{\frac{z f^{\prime \prime}(z)}{f^{\prime}(z)}+1\right\}>0$. We denote $\mathscr{C}$ be the subclass of $\mathscr{A}$ consisting of all convex functions.
T.H.Macgregor [4], investigated the properties of the class R of functions $f(z)$ which belong to $\mathscr{A}$ and satisfy $\mathfrak{R} f^{\prime}(z)>$ 0 , for $z \in \mathbb{U}$. In [3], O.P.Juneja and M.L.Mogra considered the class $\mathbf{R}(\alpha, \beta)$ of functions $f(z) \in \mathscr{A}$, satisfying the condition

$$
\left|\frac{f^{\prime}(z)-1}{f^{\prime}(z)+(1-2 \alpha)}\right|<\beta, \quad(z \in \mathbb{U})
$$

for some $\alpha, \beta(0 \leq \alpha<1, \quad 0<\beta \leq 1)$.

In 1983, Silvia [9] defined and studied the class $\mathscr{C}_{\beta}[A, B]$ of functions $f(z) \in \mathscr{A}$ satisfying

$$
\mathfrak{R}\left\{\frac{z\left(f^{\prime}(z)\right)^{\prime}}{g^{\prime}(z)}\right\}>\beta, \quad \text { where } g(z) \in \mathscr{S}^{*}[A, B], \quad(z \in \mathbb{U}) .
$$

In [7], the author introduced new subclasses of the class $\mathscr{C}_{\alpha}$ of functions $f(z)$ defined by (1) to be an alpha-close-to-convex function if $\frac{f(z) f^{\prime}(z)}{z} \neq 0$ in $\mathbb{U}$ and if for some nonnegative real number $\alpha$ and for some starlike function

$\phi(z)=z+\ldots$,

$$
\mathfrak{R}\left\{(1-\alpha) \frac{z f^{\prime}(z)}{\phi(z)}+\alpha \frac{\left(z f^{\prime}(z)\right)^{\prime}}{\phi^{\prime}(z)}\right\}>0, \quad(z \in \mathbb{U}) .
$$

In [8] the authors studied a subclass $\mathscr{C}^{\prime}$ of close to convex 
functions defined by the condition

$$
\operatorname{Re}\left(\frac{z f^{\prime}(z)}{g(z)}\right)>0, \quad \text { where } g \in \mathscr{C} .
$$

This work was further extended by Peng Zhigang [10], defining a subclass $\mathscr{C}^{\prime}(\alpha, \beta)$ by requiring that $f \in \mathscr{C}^{\prime}(\alpha, \beta)$ if and only if

$$
\left|\frac{\frac{z f^{\prime}(z)}{g(z)}-1}{\frac{z f^{\prime}(z)}{g(z)}+(1-2 \alpha)}\right|<\beta
$$

In [11], the authors studied a subclass $\mathscr{C}^{*}[A, B]$ of close to convex functions defined by the condition

$$
\begin{gathered}
\left|\frac{\left(z f^{\prime}(z)\right)^{\prime}}{g^{\prime}(z)}-1\right|<\left|A-B \frac{\left(z f^{\prime}(z)\right)^{\prime}}{g^{\prime}(z)}\right|, \text { where } g \in \mathscr{C} \\
\text { and }-1 \leq B<A \leq 1 .
\end{gathered}
$$

Let $s$ and $t$ be analytic functions in $\mathbb{U}$. Then $s$ is said to be subordinate to $t$, written $s(z) \prec t(z)$ if there is a Schwarz function $\omega(z)$ on $\mathbb{U}$ such that $\omega(0)=0$,

$|\omega(z)| \leq|z|$ for every $|z|<1$ and is such that

$$
s(z)=t(w(z)) .
$$

However it is well-known that, if $t \in \mathscr{S}$, then (2) is equivalent to $s(0)=t(0)$ and $s(\mathbb{U})=t(\mathbb{U})$.

In this paper. Motivated by the above concepts, we introduce and study

various properties of subclasses, $\mathscr{K}_{s}^{*}(\alpha, \beta)$ of close-to-star functions and

$\lambda$-close-to-star functions $\mathscr{C}_{\lambda}^{*}(\alpha, \beta)$.

Definition 1.1. A normalized regular function $f(z)$ of the form (1) is said to be in the class $\mathscr{K}_{s}^{*}(\alpha, \beta)$ if and only if there is a starlike function

$$
g(z)=z+b_{2} z^{2}+\ldots \in \mathscr{S}^{*}, \quad(z \in \mathbb{U})
$$

such that

$$
\left|\frac{\frac{z f^{\prime}(z)}{g(z)}-1}{\frac{z f^{\prime}(z)}{g(z)}+(1-2 \alpha)}\right|<\beta, \quad 0 \leq \alpha<1, \quad 0<\beta \leq 1 \quad(z \in \mathbb{U}) .
$$

Interms of subordination (2) can be put in the form $f \in$ $\mathscr{K}_{s}^{*}(\alpha, \beta)$ if and only if

$$
\frac{z f^{\prime}(z)}{g(z)} \prec \frac{1+(2 \alpha-1) \beta z}{1+\beta z} .
$$

Note that $f \in \mathscr{K}_{s}^{*}(\alpha, \beta)$ if and only if $\frac{z f^{\prime}(z)}{g(z)} \in \mathscr{P}((2 \alpha-$ 1) $\beta, \beta)$.
Definition 1.2. A normalized regular function $f(z)$ of the form (1) is said to be in the class $\mathscr{C}_{\lambda}^{*}(\alpha, \beta)$ if and only if there is a starlike function $g(z)$ defined by (3) such that

$$
(1-\lambda) \frac{f^{\prime}(z)}{g^{\prime}(z)}+\lambda \frac{\left(z f^{\prime}(z)\right)^{\prime}}{g^{\prime}(z)} \prec \frac{1+(2 \alpha-1) \beta z}{1+\beta z}, \quad(z \in \mathbb{U}),
$$

where, $0 \leq \lambda \leq 1, \quad 0 \leq \alpha<1$ and $0<\beta \leq 1$.

\section{Preliminaries}

Lemma 2.1. [1, 6] Let $N$ and $D$ be analytic in $\mathbb{U}, D$ maps onto a many-sheeted starlike region, $N(0)=0=D(0)$. Then

$$
\frac{N^{\prime}(z)}{D^{\prime}(z)} \prec \frac{1+A z}{1+B z} \Rightarrow \frac{N(z)}{D(z)} \prec \frac{1+A z}{1+B z} .
$$

\section{COEFFICIENT BOUNDS}

Theorem 3.1. If $h(z)=1+\sum_{n=1}^{\infty} h_{n} z^{n} \in p((2 \alpha-1) \beta, \beta)$, $0 \leq \alpha<1, \quad 0<\beta \leq 1, \quad z \in \mathbb{U}$, then $\left|h_{n}\right| \leq 2 \beta(1-\alpha)$, for all $n \geq 1$. This inequality is sharp.

Proof. As $h \in p((2 \alpha-1) \beta, \beta)$, we have

$$
1+\sum_{n=1}^{\infty} h_{n} z^{n} \prec \frac{1+(2 \alpha-1) \beta z}{1+\beta z}, \quad(0 \leq \alpha<1,0<\beta \leq 1) .
$$

This implies there is a Schwarz function $\omega(z)$ such that

$$
\begin{aligned}
& 1+\sum_{n=1}^{\infty} h_{n} z^{n}=\frac{1+(2 \alpha-1) \beta \omega(z)}{1+\beta \omega(z)}, \\
& \text { where } \omega(0)=0,|\omega(z)| \leq|z| \text { forall }|z|<1 .
\end{aligned}
$$

Then (6) gives,

$$
\begin{aligned}
& {\left[((2 \alpha-1) \beta-\beta)-\sum_{k=1}^{\infty} \beta h_{k} z^{k}\right]\left[\sum_{k=1}^{\infty} c_{k} z^{k}\right]=\sum_{k=1}^{\infty} h_{k} z^{k}} \\
& \text { where } \omega(z)=\sum_{n=1}^{\infty} c_{k} z^{k}
\end{aligned}
$$

Applying Cauchy's product on the left hand side of (7) and comparing

coefficients, the nth coefficient $h_{n}$ on the right hand side of (7) depends only on $h_{1}, h_{2}, \ldots, h_{n-1}$ on the left of (7).Therefore from (7), for all $n \geq 1$, it follows that

$$
\left|2(\alpha-1) \beta-\sum_{k=1}^{n-1} \beta h_{k} z^{k}\right||\omega(z)|=\sum_{k=1}^{\infty} h_{k} z^{k}+\sum_{k=n+1}^{\infty} b_{k} z^{k}
$$

where $b_{k}(k=n+1, n+2, \ldots)$ are some complex numbers. Since $|\omega(z)|<1$, we get,

$$
\begin{gathered}
\left|\sum_{k=1}^{\infty} h_{k} z^{k}+\sum_{k=n+1}^{\infty} b_{k} z^{k}\right|=\left|2(\alpha-1) \beta-\sum_{k=1}^{n-1} \beta h_{k} z^{k}\right||\omega(z)| \\
\leq\left|2(\alpha-1) \beta-\sum_{k=1}^{n-1} \beta h_{k} z^{k}\right|
\end{gathered}
$$


Squaring and integrating around the circle $z=r e^{i \theta}, \quad 0 \leq \theta \leq$ $2 \pi$,

$$
\begin{gathered}
\frac{1}{2 \pi} \int_{0}^{2 \pi}\left|2(\alpha-1) \beta-\sum_{k=1}^{n-1} \beta h_{k} r^{k} e^{i k \theta}\right|^{2} d \theta \\
\geq \frac{1}{2 \pi} \int_{0}^{2 \pi}\left|\sum_{k=1}^{n} h_{k} r^{k} e^{i k \theta}+\sum_{k=n+1}^{\infty} b_{r} r^{k} e^{i k \theta}\right|^{2} d \theta .
\end{gathered}
$$

Taking limit $r \rightarrow 1^{-}$we get,

$$
4 \beta^{2}(\alpha-1)^{2}+\sum_{k=1}^{n-1} \beta^{2}\left|h_{k}\right|^{2} \geq \sum_{k=1}^{n-1} \beta\left|h_{k}\right|^{2}+\left|h_{n}\right|^{2},
$$

which gives

$$
\left|h_{n}\right| \leq 2 \beta(1-\alpha), \quad \forall n \geq 1 .
$$

Equality is attained for the functions $h(z)=\frac{1+(2 \alpha-1) \beta z^{n}}{1+\beta z^{n}}, n=$ $2,3, \ldots$.

Theorem 3.2. If an analytic function $f$ in $\mathbb{U}$ defined by (1) satisfies the

inequality

$$
\sum_{n=2}^{\infty} n(1+\beta)\left|a_{n}\right|+\sum_{n=2}^{\infty}(1+\beta(2 \alpha-1))\left|b_{n}\right| \leq 2(1-\alpha) \beta
$$

where for $n=2,3, \ldots$, the coefficients $b_{n}$ are given by (3) then $f \in \mathscr{K}_{s}^{*}(\alpha, \beta)$.

Proof. We set for $f$ given by (1) and g defined by (3), then

$$
\begin{gathered}
M=\left|z f^{\prime}(z)-g(z)\right|-\left|\beta(1-2 \alpha) g(z)-\beta\left(z f^{\prime}(z)\right)\right| \\
=\left|\sum_{n=2}^{\infty} n a_{n} z^{n}-\sum_{n=2}^{\infty} b_{n} z^{n}\right| \\
-\left|2 \beta(1-\alpha)(z)+\beta(1-2 \alpha) \sum_{n=2}^{\infty} b_{n} z^{n}+\sum_{n=2}^{\infty} \beta n a_{n} z^{n}\right| \\
\leq \sum_{n=2}^{\infty} n\left|a_{n}\right||z|^{n}+\sum_{n=2}^{\infty}\left|b_{n}\right||z|^{n} \\
-\left[2(1-\alpha) \beta|z|-\beta(1-2 \alpha) \sum_{n=2}^{\infty}\left|b_{n}\right||z|^{n}+\sum_{n=2}^{\infty} \beta n\left|a_{n}\right||z|^{n}\right] \\
=-2(1-\alpha) \beta|z|+\sum_{n=2}^{\infty}(n(\beta+1))\left|a_{n}\right||z|^{n} \\
+\sum_{n=2}^{\infty}(1+\beta(2 \alpha-1))\left|b_{n}\right||z|^{n} .
\end{gathered}
$$

Hence for $z \in \mathbb{U}$ using (9) we have

$$
+M \leq-2(1-\alpha) \beta+\sum_{n=2}^{\infty}(n(1+\beta))\left|a_{n}\right|
$$

$$
\sum_{n=2}^{\infty}(1+\beta(2 \alpha-1))\left|b_{n}\right| \leq 0 .
$$

From the above calculation, we have

$$
\left|z f^{\prime}(z)-g(z)\right|<\left|\beta(1-2 \alpha) g(z)-\beta\left(z f^{\prime}(z)\right)\right|,
$$

Which is equivalent to the inequality (4).Hence $f \in \mathscr{K}_{s}^{*}(\alpha, \beta)$.

Theorem 3.3. Let $f(z)$ of the form (1) be in the class $\mathscr{K}_{s}^{*}(\alpha, \beta)$. Then for $z \in \mathbb{U}$

$$
\left|a_{n}\right| \leq[1-2 \beta(1-\alpha)]+(n+1)(1-\alpha) \beta .
$$

Proof. If $f \in \mathscr{K}_{s}^{*}(\alpha, \beta)$, then there is a function $g \in \mathscr{S}^{*}$ with $g(z)$ is given by (3) such that

$$
\frac{z f^{\prime}(z)}{g(z)} \in \mathscr{P}((2 \alpha-1) \beta, \beta) .
$$

Now

$$
\frac{z f^{\prime}(z)}{g(z)}=p(z) \prec \frac{1+(2 \alpha-1) \beta z}{1+\beta z} .
$$

Equating the coefficients of $z^{n}$ on both sides, we get

$$
n a_{n}=p_{n-1}+b_{2} p_{n-2}+\ldots+b_{n-1} p_{1}+b_{n} .
$$

Since $g(z) \in \mathscr{S}^{*}$, it follows that $\left|b_{n}\right| \leq n$, for all $n \geq 2$ and from Theorem 1 ,

$\left|p_{n}\right| \leq 2 \beta(1-\alpha)$ for all $n \geq 1$. Therefore, (11) yields

$$
\begin{aligned}
& \left|n a_{n}\right| \leq\left|b_{n}\right|+2 \beta(1-\alpha)\left[1+b_{2}+b_{3}+\ldots+b_{n-1}\right] \\
& \leq n+2 \beta(1-\alpha)[1+2+3+\ldots+(n-1)] .
\end{aligned}
$$

On simplifying, we get the required assertion of this theorem. Applying similar arguments, we get the following theorem.

Theorem 3.4. If the function $f(z) \in \mathscr{C}_{\lambda}^{*}(\alpha, \beta)$ is defined by (1), then

$$
\begin{aligned}
& \left|a_{n}\right| \leq \frac{1}{1+\lambda(n-1)} \\
& {\left[n(1-2 \beta(1-\alpha))+\frac{(1-\alpha) \beta(n+1)(2 n+1)}{3}\right]}
\end{aligned}
$$

where, $0 \leq \lambda \leq 1,0 \leq \alpha<1$ and $0<\beta \leq 1$.

\section{Growth and distortion theorems}

In this section, we prove the distortion and growth results for the function classes $\mathscr{K}_{s}^{*}(\alpha, \beta)$ and $\mathscr{C}_{\lambda}^{*}(\alpha, \beta)$. 
Theorem 4.1. If $f \in \mathscr{K}_{s}^{*}(\alpha, \beta), \quad 0 \leq \alpha<1$ and $0<\beta \leq$ $1|z|=r<1$, then if $\beta=1$,

$$
\frac{1-(1-2 \alpha) r}{(1+r)^{3}} \leq\left|f^{\prime}(z)\right| \leq \frac{1+(1-2 \alpha) r}{(1-r)^{3}}
$$

and

$$
\begin{gathered}
\frac{(1-\alpha) r(2+r)}{(1+r)^{2}}-\frac{(1-2 \alpha) r}{(1+r)} \\
\leq|f(z)| \leq \frac{(1-\alpha) r(2-r)}{(1-r)^{2}}-\frac{(1-2 \alpha) r}{(1-r)} .
\end{gathered}
$$

if $\beta \neq 1$,

$$
\frac{1-(1-2 \alpha) \beta r}{(1+\beta r)(1+r)^{2}} \leq\left|f^{\prime}(z)\right| \leq \frac{1+(1-2 \alpha) \beta r}{(1-\beta r)(1-r)^{2}}
$$

and

$$
\begin{gathered}
\frac{-2(1-\alpha) \beta}{(1-\beta)^{2}} \log \left(\frac{1+r}{1+\beta r}\right)+\frac{1+(1-2 \alpha) \beta}{(1-\beta)} \frac{r}{1+r} \\
\leq|f(z)| \leq \frac{2(1-\alpha) \beta}{(1-\beta)^{2}} \log \left(\frac{1-r}{1-\beta r}\right) \\
+\frac{1+(1-2 \alpha) \beta}{(1-\beta)} \frac{r}{1-r} .
\end{gathered}
$$

Proof. As $f \in \mathscr{K}_{s}^{*}(\alpha, \beta)$, there exists a function $g(z) \in \mathscr{S}^{*}$, such that

$$
z f^{\prime}(z)=p(z) g(z)
$$

where $p(z) \prec \frac{1+(2 \alpha-1) \beta z}{1+\beta z}$.

It is clear that

$$
\frac{1-(1-2 \alpha) \beta r}{1+\beta r} \leq|p(z)| \leq \frac{1+(1-2 \alpha) \beta r}{1-\beta r} .
$$

Again $g(z) \in \mathscr{S}^{*}$, then

$$
\frac{r}{(1+r)^{2}} \leq|g(z)| \leq \frac{r}{(1-r)^{2}} .
$$

Therefore,

$$
\frac{1-(1-2 \alpha) \beta r}{(1+\beta r)(1+r)^{2}} \leq\left|f^{\prime}(z)\right| \leq \frac{1+(1-2 \alpha) \beta r}{(1-\beta r)(1-r)^{2}} .
$$

For $z=r e^{i \theta}$, with $0<r<1$, we have

$$
f(z)=\int_{0}^{r} f^{\prime}\left(t e^{i \theta}\right) e^{i \theta} d t .
$$

From (13) and (14) we get

$$
f(z)=\int_{0}^{r} f^{\prime}\left(t e^{i \theta}\right) e^{i \theta} d t \leq \int_{0}^{r} \frac{1+(1-2 \alpha) \beta t}{(1-\beta t)(1-t)^{2}} d t .
$$

If $\beta=1$, then

$$
|f(z)| \leq \frac{(1-\alpha) r(2-r)}{(1-r)^{2}}-\frac{(1-2 \alpha) r}{(1-r)} .
$$

If $\beta \neq 1$, then

$$
|f(z)| \leq \frac{2(1-\alpha) \beta}{(1-\beta)^{2}} \log \left(\frac{1-r}{1-\beta r}\right)+\frac{1+(1-2 \alpha) \beta}{(1-\beta)} \frac{r}{1-r} .
$$

To prove the lower bound of $|f(z)|$ we proceed as follows. Let $\sigma$ be the radius of the open disc contained in the map of $\mathbb{D}$ by $f(z)$. Let $z_{0}$ be the point of $|z|=r$ for which $|f(z)|$ attains its minimum value. This minimum increases with $r$ and is less than $\sigma$. Hence, the linear segment $\Gamma$ connecting the origin with the point $f\left(z_{0}\right)$ will be covered entirely by the values of $f(z)$ in $\mathbb{D}$. Let $\gamma$ be the arc in $\mathbb{D}$ which is mapped by $\omega=f(z)$ onto this linear segment. Then

$$
\begin{aligned}
& \left|f\left(z_{0}\right)\right|=\int_{\Gamma}|d \omega|=\int_{\Gamma}\left|f^{\prime}(z) d z\right| \\
& \quad \geq \int_{\Gamma}\left|f^{\prime}(z)\right||d z| \geq \int_{0}^{r} \frac{1-(1-2 \alpha) \beta t}{(1+\beta t)(1+t)^{2}} d t .
\end{aligned}
$$

If $\beta=1$, then

$$
|f(z)| \geq \frac{(1-\alpha) r(2+r)}{(1+r)^{2}}-\frac{(1-2 \alpha) r}{(1+r)} .
$$

If $\beta \neq 1$, then

$$
|f(z)| \geq \frac{-2(1-\alpha) \beta}{(1-\beta)^{2}} \log \left(\frac{1+r}{1+\beta r}\right)+\frac{1+(1-2 \alpha) \beta}{(1-\beta)} \frac{r}{1+r} .
$$

Theorem 4.2. Let $0 \leq \lambda \leq 1,0 \leq \alpha<1,0<\beta \leq 1$ and $f \in \mathscr{C}_{\lambda}^{*}(\alpha, \beta)$.

(1) If $\lambda=0$, then for $|z|=r<1$, we have

$$
\begin{gathered}
\int_{0}^{r} \frac{1-(1-2 \alpha) \beta t}{(1+\beta t)} \frac{(1-t)}{(1+t)^{3}} d t \leq|f(z)| \\
\quad \leq \int_{0}^{r} \frac{1+(1-2 \alpha) \beta t}{(1-\beta t)} \frac{(1+t)}{(1-t)^{3}} d t .
\end{gathered}
$$

which gives

$$
\mathscr{L}_{1} \leq|f(z)| \leq \mathscr{L}_{2},
$$

where

$$
\begin{gathered}
\mathscr{L}_{1}=\frac{2 \beta(1-\alpha)(1+\beta)}{(1-\beta)^{3} r} \log \left(\frac{1+r}{1+\beta r}\right) \\
+\frac{(1-2 \alpha) \beta^{2}+2 \beta(3 \alpha-2)-1}{(1-\beta)^{2}} \frac{1}{1+r} \\
+\frac{[1+(1-2 \alpha) \beta]}{(1-\beta)} \frac{r+2}{(1+r)^{2}},
\end{gathered}
$$

and

$$
\begin{gathered}
\mathscr{L}_{2}=\frac{-2 \beta(1-\alpha)(1+\beta)}{(1-\beta)^{3} r} \log \left(\frac{1-r}{1-\beta r}\right) \\
+\frac{(1-2 \alpha) \beta^{2}+2 \beta(3 \alpha-2)-1}{(1-\beta)^{2}} \frac{1}{1-r}
\end{gathered}
$$




$$
-\frac{2[1+(1-2 \alpha) \beta]}{(1-\beta)} \frac{r-2}{(1-r)^{2}} .
$$

(2)If $0<\lambda \leq 1$, then for $|z|=r<1$, we have

$$
\mathscr{M}_{1} \leq|f(z)| \leq \mathscr{M}_{2}
$$

where

$$
\begin{aligned}
& \mathscr{M}_{1}=\frac{1}{\lambda} r^{1-\frac{1}{\lambda}} \int_{0}^{r} \int_{0}^{s} \frac{1-(1-2 \alpha) \beta t}{(1+\beta t)} \frac{1-t}{(1+t)^{3}} d t s^{\frac{1}{\lambda}-2} d s . \\
& \mathscr{M}_{2}=\frac{1}{\lambda} r^{1-\frac{1}{\lambda}} \int_{0}^{r} \int_{0}^{s} \frac{1+(1-2 \alpha) \beta t}{(1-\beta t)} \frac{1+t}{(1-t)^{3}} d t s^{\frac{1}{\lambda}-2} d s .
\end{aligned}
$$

Proof. Suppose that $f \in \mathscr{C}_{\lambda}^{*}(\alpha, \beta)$, there exists a function $g(z) \in \mathscr{S}^{*}$,

such that $(1-\lambda) f^{\prime}(z)+\lambda\left(z f^{\prime}(z)\right)^{\prime}=g^{\prime}(z) h(z)$, where

$$
h(z)=\frac{1+(2 \alpha-1) \beta z}{1+\beta z} .
$$

It is clear to see that then

$$
\frac{1-r}{(1+r)^{3}} \leq\left|g^{\prime}(z)\right| \leq \frac{1+r}{(1-r)^{3}} .
$$

Setting $F^{\prime}(z)=(1-\alpha) f^{\prime}(z)+\alpha\left(z f^{\prime}(z)\right)^{\prime}$, we have

$$
\begin{aligned}
& \frac{1-(1-2 \alpha) \beta r}{(1+\beta r)} \frac{1-r}{(1+r)^{3}} \leq\left|F^{\prime}(z)\right| \\
& ; \leq \frac{1+(1-2 \alpha) \beta r}{(1-\beta r)} \frac{1+r}{(1-r)^{3}} .
\end{aligned}
$$

Therefore,

$$
\begin{aligned}
& \int_{0}^{r} \frac{1-(1-2 \alpha) \beta t}{(1+\beta t)} \frac{1-t}{(1+t)^{3}} d t \\
& \quad \leq|F(z)| \leq \int_{0}^{r} \frac{1+(1-2 \alpha) \beta t}{(1-\beta t)} \frac{1+t}{(1-t)^{3}} d t . \\
& \int_{0}^{r} \frac{1-(1-2 \alpha) \beta t}{(1+\beta t)} \frac{1-t}{(1+t)^{3}} d t \\
& \quad \leq\left|(1-\lambda) f(z)+\lambda z f^{\prime}(z)\right| \\
& \quad \leq \int_{0}^{r} \frac{1+(1-2 \alpha) \beta t}{(1-\beta t)} \frac{1+t}{(1-t)^{3}} d t .
\end{aligned}
$$

Consider the following two cases:

(1) When $\lambda=0$. From (20) and using simple computation, we get (16).

(2) When $0<\lambda \leq 1$, using a simple computation from (20), we get (17).

Theorem 4.3. Let $f(z) \in \mathscr{K}_{s}^{*}(\alpha, \beta), 0 \leq \alpha<1,0<\beta \leq 1$. Then

$$
\operatorname{Re}\left\{\frac{z f^{\prime}(z)}{g(z)}\right\} \geq \mathscr{M}
$$

$$
\begin{aligned}
& \text { where } \\
& \mathscr{M}= \begin{cases}\frac{1-r}{1+r}-\frac{2(1-\alpha) \beta r}{(1+\beta r)[1+(2 \alpha-1) \beta r]}, & \text { if } r_{1} \leq r_{2}, \\
\frac{1-r}{1+r}+\gamma-\frac{\alpha}{1-\alpha}, & \text { if } r_{1} \geq r_{2} .\end{cases} \\
& \text { and }
\end{aligned}
$$

$$
\begin{aligned}
7 \gamma=\frac{\sqrt{(1+\beta)[1+(2 \alpha-1) \beta]\left(1-\beta r^{2}\right)\left[1-(2 \alpha-1) \beta r^{2}\right]}}{(1-\alpha) \beta\left(1-r^{2}\right)} \\
\\
\quad \frac{-\left[1+(1-2 \alpha) \beta^{2} r^{2}\right]}{(1-\alpha) \beta\left(1-r^{2}\right)}
\end{aligned}
$$

where $r_{1}$ and $r_{2}$ are the unique roots of the equations

$$
\begin{gathered}
(2 \alpha-1) \beta^{3} r^{3}-\beta[(2 \alpha-1) \beta-1] r^{2} \\
-[2(2 \alpha-1) \beta-1] r-1=0
\end{gathered}
$$

and

$$
\begin{aligned}
& {[1-(1-2 \alpha) \beta] \beta r^{4}-2[1-(1-2 \alpha) \beta] \beta r^{3}} \\
& -\{(1-\beta)[1-(1-2 \alpha) \beta] \\
& +2 \beta(\alpha-1)\} r^{2}+2[1-(1-2 \alpha) \beta] r \\
& -[1-(1-2 \alpha) \beta]=0
\end{aligned}
$$

respectively.

Proof. Since $f(z) \in \mathscr{K}_{s}^{*}(\alpha, \beta)$, we can write

$$
\frac{z f^{\prime}(z)}{g(z)}=p(z) \prec \frac{1+(2 \alpha-1) \beta z}{1+\beta z} \text { where } g(z) \in \mathscr{S}^{*} \text {. }
$$

Hence $F(z)=z f^{\prime}(z)$ and therefore

$$
\frac{z F^{\prime}(z)}{F(z)}=\frac{z g^{\prime}(z)}{g(z)}+\frac{z p^{\prime}(z)}{p(z)} \text {. }
$$

For starlike function $g(z)$ of order $\alpha(0 \leq \alpha<1)$, we have

$$
\operatorname{Re}\left\{\frac{z g^{\prime}(z)}{g(z)}\right\} \geq \frac{1-r}{1+r} .
$$

As $p(z) \prec \frac{1+(2 \alpha-1) \beta z}{1+\beta z}$, we have [2],

$$
\operatorname{Re}\left\{\frac{z p^{\prime}(z)}{p(z)}\right\} \geq \begin{cases}\frac{-2(1-\alpha) \beta r}{(1+\beta r)[1+(2 \alpha-1) \beta r]}, & \text { if } r_{1} \leq r_{2} \\ \gamma-\frac{\alpha}{1-\alpha}, & \text { if } r_{1} \geq r_{2} .\end{cases}
$$

where

$$
\begin{aligned}
7 \gamma=\frac{\sqrt{(1+\beta)[1+(2 \alpha-1) \beta]\left(1-\beta r^{2}\right)\left[1-(2 \alpha-1) \beta r^{2}\right]}}{(1-\alpha) \beta\left(1-r^{2}\right)} \\
\\
\frac{-\left[1+(1-2 \alpha) \beta^{2} r^{2}\right]}{(1-\alpha) \beta\left(1-r^{2}\right)}
\end{aligned}
$$

and $r_{1}, r_{2}$ are the unique roots of the equation

$$
\begin{aligned}
& (2 \alpha-1) \beta^{2} r^{4}-2(2 \alpha-1) \beta^{2} r^{3} \\
& \quad-\left[1+4 \alpha \beta+(2 \alpha-1) \beta^{2}\right] r^{2}-2 r+1=0
\end{aligned}
$$

in the interval $(0,1]$.From (24) (25) and (26), we get the required result. 


\section{References}

[1] Aghalary,R and Kulkarni, S.R. (2001): Some properties of the integral operators in univalent function, Studia Univ.Babes,-Bolyai Math., 46 no. 1, 3- 10.

[2] Janowski, W. (1973): Some extremal problems for certain families of analytic functions, I. Ann. Polon. Math., 28 297-326.

[3] Juneja, Om Prakash, Mogra and Manak Lal. (1979):A class of univalent functions, Bull. Sci. Math., (2) 103 no. 4, 435-447.

[4] MacGregor, T.H. (1962): Functions whose derivative has a positive real part, Trans Amer Math Soc., 104 532-537.

[5] Noor, K.I.(1987):On quasi-convex functions ad related topics, Inter. J. Math. Math. 10 241-258.

[6] Parvatham,R and Shanmugam,T.N.(1983): On analytic functions with reference to integral operator, Bull. Austral. Math. Soc., 28 no. 2, 207-215.

[7] Pran Nath Chichra,(1977): New subclasses of the class of close to convex functions, Proc. of the Amer. Math. Soc., 62 no. 1, 37-43.

[8] Selvaraj,C.(2004): A subclass of close-to-convex functions, Southeast Asian Bull.Math., 28 no. 1, 113-123.

[9] Silvia,E.M. (1983): Subclasses of close-to-convex functions, Inter. J. Math. Sci., $6449-458$.

[10] Peng and Zhigang.(2010) : On a subclass of close-toconvex functions, Acta Math. Sci.ser. B Engl. Ed., 30 no. 5, 1449-1456.

[11] Xiong, Liangpeng; Liu, Xiaoil,(2012):A general subclass of close-to-convex functions, Kragujevac J. Math.,36 no.2, 215-260.

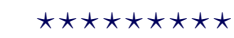

ISSN(P):2319- 3786

Malaya Journal of Matematik

ISSN(O):2321 - 5666

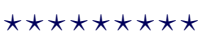

